The University of Southern Mississippi

The Aquila Digital Community

Faculty Publications

$1-1-2012$

\title{
Willingness to Use ADHD Treatments: A Mixed Methods Study of Perceptions by Adolescents, Parents, Health Professionals and Teachers
}

\author{
Regina Bussing \\ University of Florida, rbussing@ufl.edu \\ Mirka Koro-Ljungberg \\ University of Florida, mirka.koro-ljunberg@asu.edu \\ Kenji Noguchi \\ University of Southern Mississippi, kenji.noguchi@usm.edu \\ Dana Mason \\ University of Florida, dmason@ufl.edu \\ Gillian Mayerson \\ University of Florida
}

See next page for additional authors

Follow this and additional works at: https://aquila.usm.edu/fac_pubs

Part of the Psychology Commons

\section{Recommended Citation}

Bussing, R., Koro-Ljungberg, M., Noguchi, K., Mason, D., Mayerson, G., Garvan, C. W. (2012). Willingness to Use ADHD Treatments: A Mixed Methods Study of Perceptions by Adolescents, Parents, Health Professionals and Teachers. Social Science \& Medicine, 74(1), 92-100.

Available at: https://aquila.usm.edu/fac_pubs/275

This Article is brought to you for free and open access by The Aquila Digital Community. It has been accepted for inclusion in Faculty Publications by an authorized administrator of The Aquila Digital Community. For more information, please contact Joshua.Cromwell@usm.edu. 


\section{Authors}

Regina Bussing, Mirka Koro-Ljungberg, Kenji Noguchi, Dana Mason, Gillian Mayerson, and Cynthia W. Garvan 
Published in final edited form as:

Soc Sci Med. 2012 January ; 74(1): 92-100. doi:10.1016/j.socscimed.2011.10.009.

\title{
Willingness to use ADHD Treatments: A Mixed Methods Study of Perceptions by Adolescents, Parents, Health Professionals and Teachers
}

\author{
Regina Bussing ${ }^{\mathrm{a}}$, Mirka Koro-Ljungberg ${ }^{\mathrm{b}}$, Kenji Noguchi ${ }^{\mathrm{c}}$, Dana Mason ${ }^{\mathrm{a}}$, Gillian \\ Mayerson ${ }^{\mathrm{a}}$, and Cynthia W Garvan ${ }^{\mathrm{b}}$ \\ aDepartment of Psychiatry, University of Florida, Gainesville, FI \\ bSchool of Human Development and Organizational Studies in Education, University of Florida, \\ Gainesville, FI \\ 'Department of Psychology, University of Southern Mississippi Gulf Coast, Long Beach, MS
}

\begin{abstract}
Little is known about factors that influence willingness to engage in treatment for attention deficit/ hyperactivity disorder (ADHD). From 2007 to 2008, in the context of a longitudinal study assessing ADHD detection and service use in the United States, we simultaneously elicited ADHD treatment perceptions from four stakeholder groups: adolescents, parents, health care professionals and teachers. We assessed their willingness to use ADHD interventions and views of potential undesirable effects of two pharmacological (short- and long-acting ADHD medications) and three psychosocial (ADHD education, behavior therapy, and counseling) treatments. In multiple regression analysis, willingness was found to be significantly related to respondent type (lower for adolescents than adults), feeling knowledgeable, and considering treatments acceptable and helpful, but not significantly associated with stigma/embarrassment, respondent race, gender and socioeconomic status. Because conceptual models of undesirable effects are underdeveloped, we used grounded theory method to analyze open-ended survey responses to the question: "What other undesirable effects are you concerned about?" We identified general negative treatment perceptions (dislike, burden, perceived ineffectiveness) and specific undesirable effect expectations (physiological and psychological side-effects, stigma and future dependence on drugs or therapies) for pharmacological and psychosocial treatments. In summary, findings indicate significant discrepancies between teens' and adults' willingness to use common ADHD interventions, with low teen willingness for any treatments. Results highlight the need to develop better treatment engagement practices for adolescents with ADHD.
\end{abstract}

\section{Keywords}

Attention Deficit Hyperactivity Disorder; treatment willingness; stakeholder perceptions; side effect perceptions; barriers to evidence-based treatment; USA

\footnotetext{
(C) 2011 Elsevier Ltd. All rights reserved.

Corresponding author: Regina Bussing rbussing@ufl.edu.
}

Publisher's Disclaimer: This is a PDF file of an unedited manuscript that has been accepted for publication. As a service to our customers we are providing this early version of the manuscript. The manuscript will undergo copyediting, typesetting, and review of the resulting proof before it is published in its final citable form. Please note that during the production process errors may be discovered which could affect the content, and all legal disclaimers that apply to the journal pertain. 


\section{Introduction}

Treatments for attention deficit/hyperactivity disorder (ADHD), a common neurodevelopmental disorder characterized by the presence of impairing levels of inattention, hyperactivity and impulsivity (American Psychiatric Association, 2000), have considerable public health and economic relevance in the United States, Canada, Europe and other industrialized countries (Polanczyk et al., 2007). ADHD treatments have been the focus of extensive scientific study as well as of public controversy, in part due to concerns over steadily increasing prescriptions of methylphenidate and other psychostimulants for those diagnosed with ADHD (Rey \& Sawyer, 2003). The efficacy of medication treatment for ADHD symptom reduction has been sufficiently documented to make the medications a mainstay of professional treatment parameters and guidelines (American Academy of Child \& Adolescent Psychiatry \& Work Group on Quality Issues, 2002; American Academy of Pediatrics, 2001). Furthermore, behavioral parent training and behavioral contingency management in school settings, but not office-based psychotherapies conducted solely with the child and sometimes referred to generically as "counseling", have been identified as well-established psychosocial treatments for ADHD (Pelham, Jr. \& Fabiano, 2008).

Despite efforts to increase the use of "scientifically proven" or evidence-based treatments" within usual care settings, U.S. surveys of physician practices indicated that only $50 \%$ of children with ADHD were receiving care corresponding to practice parameters of the American Academy of Child and Adolescent Psychiatry (Hoagwood et al., 2000). Important factors that may influence the use of evidence-based practices are social perceptions (e.g., like/dislike, perceived effectiveness, anticipated stigma) of affected youth, their parents, health professionals, and teachers in regards to treatment acceptability, and treatment-related risks and benefits.

Few studies have examined youth perceptions of ADHD treatment and conclusions are limited by small sample sizes and convenience sampling. Studies report that ADHD-treated youth perceived positive effects of stimulant medications on attention, behavior and social function, yet, less than one-half wanted to continue taking medication (Moline \& Frankenberger, 2001), perhaps due to their experiences of high levels of medication side effects (dosReis et al., 2003). Furthermore, youth did not find medication as beneficial as their parents (McNeal et al., 2000). Studies of parent ADHD treatment acceptability have consistently found higher ratings for behavioral than for medication interventions (Johnston et al., 2008; Krain et al., 2005). Parents of children with ADHD rate medication treatments as more effective than parents without personal experiences (Gage \& Wilson, 2000; Stroh et al., 2008). According to Gage (2000), parents raising a child with ADHD were more likely to support a multimodal approach to treatment than parents without the personal experience. Caucasian parents have consistently been found to be more accepting of ADHD medications than non-Caucasian parents (dosReis et al., 2003; Krain et al., 2005). In a study conducted by dosReis (2003), non-Caucasian parents expressed concerns that medication use may lead to drug abuse and has other extremely negative side effects (dosReis et al., 2003).

Teachers serve as information sources for parents looking for advice related to ADHD, so teacher perceptions of ADHD treatments may also influence access to evidence-based treatments. Similar to parents, teachers have generally rated behavioral interventions as more acceptable than medication treatments (Curtis et al., 2006; Vereb \& DiPerna, 2004). Studies seeking to identify predictors of teacher treatment acceptability reported absent (Power et al., 1995) or small (Vereb \& DiPerna, 2004) relationships to ADHD training or years of teaching experience, and possible influences of the affected students' genders, suggesting that teachers may view girls as better candidates for behavioral interventions than for medications (Curtis et al., 2006). 
Health professionals' perceptions of ADHD treatment are a particularly under researched area. A study in Kansas reported that school nurses were generally knowledgeable and supportive of medication as a form of treatment, however, they maintained a personal skepticism about medication safety and use (Frisch et al., 2003). In a mail survey of physicians, psychiatrists were more concerned about side effects, including sleep problems, decreased appetite or weight loss, than family doctors, but both groups of prescribers indicated that they thought stimulant medications effective in treating ADHD symptoms (Stockl et al., 2003). Of note, in recent years various long-acting ADHD medications have become available which do not need to be administered during school hours, thereby eliminating potential barriers to treatment. However, existing ADHD treatment acceptability studies do not specifically address perceptions surrounding long-acting medications.

To complement findings derived from quantitative research methods, several qualitative studies have focused on perceived benefits and side effects of ADHD medications.

According to Charach and colleagues (2006), parents of children with ADHD balance the costs and benefits of using medication. Diverse factors, such as a child's dislike of taking pills, stigma experiences, and lowered self-esteem, contributed to the discontinuation of medication, even when clear symptom reductions had occurred (Charach et al., 2006). Similar findings about parents weighing benefits against side-effects were reported by Hansen and Hansen (2006). Academic improvements were listed as one reason to tolerate negative side-effects of medication (Hansen \& Hansen, 2006).

The current research seeks to address gaps in our knowledge base by simultaneously eliciting ADHD treatment perceptions from four stakeholders: adolescents, parents, health care professionals and teachers. Specifically, the study assessed participants' willingness to use ADHD interventions and views of acceptability, effectiveness, and potential side effects associated with treatment. We used a mixed methods approach, combining qualitative and quantitative research methods that informed each other and finally led to the development of the survey instrument described in this paper. Mixed methods were chosen because our understanding of treatment concerns is still underdeveloped, without clearly established measurement approaches, and because we wanted to contribute differently constructed knowledge about concerns elicited by pharmacological and psychosocial ADHD treatments. The study sought to answer the following research questions:

1. How divergent are adolescents, parents, health professionals and teachers in their willingness to use or recommend the use of ADHD pharmacological (short- and long-acting medications) and psychosocial (ADHD education, behavior therapy, and counseling) treatments)? We hypothesized that adolescents express lower willingness than adult respondents for any ADHD interventions, and that health professionals are more willing to use pharmacological treatments than the other three respondent groups.

2. What demographic, perceptual and experiential factors predict willingness to use pharmacological or psychosocial ADHD treatments? We hypothesized that Caucasian respondents express higher willingness than African American respondents to use medication interventions. We further hypothesized that ADHD treatment willingness is increased by higher perceptions of acceptability and effectiveness and lowered by expectations of side effects and embarrassment. Informed by research on parental medication receptivity we hypothesized that past ADHD treatment experiences increases willingness to use ADHD medications.

3. How do concerns about side-effect influence adolescents, parents, teachers, and health professionals' perceptions about the use of empirically supported ADHD treatments? 


\section{Methods}

\section{Participants}

The parents and adolescent participants for this study were part of a longitudinal mixed methods study of ADHD detection and service use in the United States (2007-2008) and included cohort members of our ADHD high-risk group and of the low-risk peer group (Bussing et al., 2010). Study participants originally were derived from stratified random sampling of public school records, oversampling girls by a factor of 2 to ensure sufficient representation. Parents of 1,615 elementary school students completed telephone screening interviews and teachers made behavior ratings of 1,205 students. Children were assigned to the ADHD risk group if they were previously diagnosed with ADHD, specifically suspected of having ADHD, or obtained elevated parent and teacher ADHD behavior ratings. The ADHD low-risk peers were participants without prior ADHD diagnosis or concerns whose behavior ratings were in the normal range. The study setting was a school district in North Florida containing an urban center and several smaller communities with rural characteristics. Teachers were randomly selected from the local school district staff database. Health professionals consisted of two groups of participants. The first group consisted of school health professionals, including school nurses, psychologists and guidance counselors, who, like the teachers, were randomly selected from the local school district staff database. The second group of health professionals represented participating high-risk adolescents' current or past ADHD treatment providers who had been identified during our service assessment interviews and included pediatricians, social workers, psychologists and child psychiatrists.

\section{Procedures}

The Institutional Review Board at the University of Florida approved this study. Parental consent and child assent were obtained for all surveys completed by adolescents. Written informed consent was waived for all adult participants; instead, completion of the survey constituted consent. Participants received the survey in the mail, completed it, and then mailed it back. Parent and adolescent participants also had the option of completing the survey questionnaire during a study visit conducted at a location of their choice, including home, research office or community library. To increase participation rates, participants received reminder phone calls if the survey had not been mailed back after three weeks from the date originally mailed. If the participant indicated that they had not received the survey or had lost/misplaced it, the address was verified and another copy was mailed out. This procedure was repeated two weeks after the mailing of the second copy. Respondents received a $\$ 15$ gift card incentive upon survey completion.

\section{Development of ADHD Treatment Perception Survey}

Items for the treatment perception survey were developed through: 1) two qualitative data collection phases using experience sampling and focus groups (Williamson et al., 2009); 2) reviews of extant literature including practice guidelines and practice parameters (American Academy of Child and Adolescent Psychiatry, 2002; American Academy of Pediatrics, 2001; Pelham \& Fabiano, 2008); and 3) pilot testing of item wording, response formats and vignettes. The five treatment approaches reported in this manuscript included four empirically supported ADHD interventions, namely ADHD education, short-acting stimulant medication, long-acting stimulant medication, and behavior therapy (American Academy of Child and Adolescent Psychiatry, 2002; American Academy of Pediatrics, 2001), as well as an unsupported treatment, counseling therapy (Pelham \& Fabiano, 2008). We included counseling therapy because it was repeatedly referred to as helpful by participants in our qualitative research. To anchor survey responses, we developed a vignette describing a child with sufficient DSM-IV symptoms to qualify for an ADHD diagnosis, and 
respondents answered questions regarding the person in the vignette, which had male and female versions (fictitious names chosen were "Jennifer" and "Joseph").

\section{Measures}

Survey items-The five treatment approaches were described as follows (using the example of a female vignette): 1) short-acting medication: "Jennifer" takes short-acting ADHD medication several times daily, including at school; 2) long-acting medication: "Jennifer" takes long-acting ADHD medication every morning, in private at home; 3) behavior therapy: Parents receive professional instruction about behavior therapy strategies, like using rewards and consequences, to improve "Jennifer's" ADHD behavior problems; 4) ADHD education: "Jennifer" and family receive education from their treatment provider about ADHD, its causes, symptoms, and possible treatments; and 5) counseling therapy: "Jennifer" gets individual therapy (counseling). After reading the vignette, respondents rated each of the five treatments on a 5-point Likert scale $(1=$ not at all, $3=$ moderately, $5=$ very much) for acceptability, perceived effectiveness/helpfulness, potential to be embarrassing (intended to represent a proxy for treatment stigma), likelihood to cause undesirable effects, and their self-rated level of knowledge about the treatment. Respondents then rated their willingness to use a given treatment or recommend its use ( $1=$ not willing at all, $3=$ moderately willing, $5=$ very willing), and willingness to use each of the five interventions served as dependent variables for our quantitative analyses.

Open-ended question-Embarrassment ratings were followed by the open-ended question "What other undesirable effects are you concerned about?" and respondents could write about any other effects they attributed to the treatment. Responses were transcribed and utilized for the qualitative data analysis.

Sociodemographics-Information about respondent gender, race, age and socioeconomic status (SES) was obtained through survey questionnaires. SES scores were calculated using the Hollingshead 4-factor Index (Hollingshead, 1975).

Past ADHD treatment experience-Receipt of ADHD treatment services was assessed using the Child and Adolescent Services Assessment (CASA) (Burns et al., 1992), a parentreport measure that inquires about mental health service use from 33 treatment settings including inpatient, outpatient and informal care. Validity studies have shown good to excellent agreement between CASA parent reports and medical records for receiving outpatient services (Ascher et al., 1996; Bussing, Mason et al., 2003), and excellent agreement on details of the child's medication regimens.

\section{Variable construction and statistical analysis}

Our original dependent variables consisted of the five willingness ratings mentioned above; to be consistent with extant literature on treatment acceptability we also constructed composite willingness variables for medications (combining ratings of short- and longacting medications) and for psychosocial treatments (combining ratings of behavior therapy, psychoeducation, and counseling).

To address Aim 1 we conducted a Kruskal-Wallis for each of the five dependent variables (i.e., ADHD treatment willingness) by our main independent predictor variable (i.e., respondent type) followed by Bonferroni corrected Wilcoxon rank sum tests. We constructed box plots to display and compare the willingness data by respondent type. Box plots conventionally display the median (solid line) and mean (cross), boxes depicting the lower (Q1) and upper (Q3) quartile, whiskers (minimum and maximum values within inner fences, defined as Q1, Q3 -/+, 1.5* interquartile range), and identify outliers (open circle) 
that fall outside the inner fences. Aim 2 was addressed using a multiple regression analysis for each of the composite dependent variables (willingness to use medications and willingness to use psychosocial treatments). Our independent variables were potentially mutable perceptual variables (feeling knowledgeable, treatment acceptability, effectiveness, side effects, potential embarrassment). We adjusted for respondent type and for other potential confounding respondent sociodemographic predictors (age, race, SES, gender). To address how past treatment experience predicted willingness, we conducted an ANOVA for each of the composite dependent variables by an independent three-level variable that distinguished ADHD risk status and prior ADHD treatment experience (ADHD high-risk treated [Risk-TX], ADHD high-risk untreated [Risk-UNTX]; ADHD low-risk peers untreated [Peer-UNTX]). The Tukey-Kramer multiple comparison procedure was then applied. Our level of significance was set at .05; all hypothesis testing was two-sided. Analyses were performed using SAS (version 9.2; Cary, N.C.).

Qualitative analysis methods: Please see integrated qualitative methods and findings section

\section{Results}

The survey was completed by 569 participants consisting of 161 parents (28\%), 148 adolescents (26\%), 48 ADHD treatment providers (8\%), 90 school health professionals (16\%), and 122 school district teachers (21\%). Response rates ranged from 50\% (teachers) to $67 \%$ (school professionals), refusal rates from $10 \%$ (school professionals) to $19 \%$ (teachers), and the remaining nonparticipants were unreachable or failed to return surveys without expressing explicit refusal. Further detail about participants' sociodemographic and other characteristics is provided in Table 1. Adolescent age ranged from 14 years to 19 years (16.5 years; SD 1.3 years). The adult participants consisted mostly of females, and more parents than health professionals or teachers were in the 41-50 year age range. Consistent with participant differences in educational backgrounds and professional status, Hollingshead scores were higher for health professionals and teachers than for adolescent and parent participants. Teachers and health professionals reported on average similar length of professional experience. Of the 148 adolescents 85 (57\%) were ADHD high risk with previous ADHD treatment (Risk-TX), 25 (17\%) were untreated ADHD high-risk (RiskUNTX), and 38 (26\%) were low-risk peers without ADHD treatment experience (PeerUNTX). The corresponding parent numbers and percentages were 94 (58\%), $28(17 \%)$ and 39 (24\%). ADHD symptoms varied significantly by risk and previous treatment status, $F$ $(2,144)=21.42, p<.0001$. Previously treated and untreated adolescents in the high-risk group exhibited on average $1.14(S D 0.72)$ and 0.79 (SD 0.58), respectively, ADHD symptoms per parent report on the Vanderbilt ADHD Diagnostic Parent Rating Scale, whereas peers in the low risk group exhibited 0.35 ( $S D 0.37$ ) symptoms. Pairwise comparison using Tukey-Kramer showed significant differences for all contrasts.

\section{Quantitative Findings}

Respondent willingness to use treatments-Each of the five Kruskal-Wallis analyses was significant with all $p$-values less than .0001. As hypothesized, we found that adolescents expressed significantly lower willingness than adult respondents for all ADHD interventions. The other three respondent groups had similar responses for short- and longacting ADHD medication and for behavior therapy. We found a significant difference between teachers and health professionals for ADHD education interventions with health professionals indicating a higher willingness towards professionally rendered ADHD education than teachers. Both parents and health professionals indicated a significantly higher willingness to utilize/recommend utilizing counseling than teachers. Box plots for the five treatment willingness variables are displayed in Graphs 1 and 2. 
Predictors of treatment willingness-Our multiple regression prediction model of medication willingness was statistically significant, $F(12,539)=35.86, p<.0001$, and explained a considerable amount of response variance $(R$-Square $=0.44)$. Regression coefficients (betas) are given in Table 2. As shown in more detail in Table 2, of the hypothesized perceptual predictors only embarrassment was not significantly associated with willingness to use medications for ADHD. Willingness to use pharmacological ADHD treatment was increased by feeling knowledgeable, and by considering medications acceptable and helpful (beta estimates of $0.22,0.47$ and 0.24 , respectively), but was reduced by anticipation of negative side effects (beta estimate of -0.11). Respondent type remained significantly associated with willingness to use ADHD medications even after controlling for perceptual variables and sociodemographic characteristics, such that parents and health professionals expressed significantly higher willingness than adolescents (beta estimates of 0.55 and 0.44 , respectively). Results of multiple comparison testing, using the TukeyKramer adjustment, failed to show differences in parents' and health professionals' medication willingness $(\mathrm{M}=3.17$ versus 3.06; $p=.79)$, and in adolescent and teacher medication willingness ( $\mathrm{M}=2.62$ versus $2.76 ; p=.72)$; multiple comparison testing further demonstrated significant differences between medication willingness of parents and teachers $(\mathrm{M}=3.17$ versus $2.76, p=.0041)$, parents and adolescents $(\mathrm{M}=3.17$ versus $2.62, p<.0001)$, health professionals and teachers ( $\mathrm{M}=3.06$ versus $2.76, p=.0445)$, and health professionals and adolescents $(\mathrm{M}=3.06$ versus $2.62, p<.0032)$. In the multiple regression analysis, respondent race, gender and SES did not remain independently associated with willingness to use ADHD medications.

Similar findings apply to willingness to use psychosocial treatments, with a multiple regression prediction model that was statistically significant, $F(12,541)=31.11, p<.0001$, and explained a considerable amount of response variance $(R-$ Square $=0.59)$. Like medication use, willingness to use psychosocial ADHD treatment was increased by feeling knowledgeable and by considering these interventions acceptable and helpful (beta estimates of $0.27,0.44$ and 0.19 , respectively). Anticipation of negative side effects reduced willingness to use psychosocial treatments (beta estimate of -0.15). Respondent type also remained significantly associated with willingness to use psychosocial treatments for ADHD after controlling for perceptual variables and sociodemographic characteristics, such that parents, health professionals, and teachers expressed significantly higher willingness than adolescents (beta estimates of $0.64,0.46$ and 0.46 , respectively). Results of multiple comparison testing, using the Tukey-Kramer adjustment, failed to show differences among parents', health professionals' and teachers' willingness to use psychosocial interventions for $\operatorname{ADHD}(\mathrm{M}=3.72,3.54$ and 3.55, respectively). Sociodemographic characteristics were not independently associated with willingness to use psychosocial treatments in the multiple regression analysis.

Examination whether composite treatment willingness varied by our three-level ADHD treatment experience/cohort risk variable (Risk-TX, Risk-UNTX, Peer-UNTX) revealed no differences for adolescents in medication $(M(S D)$ of 2.6 (1.33), 2.0 (1.17), 2.4 (1.17), respectively; $F(2,144)=2.13, p=0.13)$ or psychosocial treatment willingness $(M(S D)$ of 2.7 (1.04), $2.7(1.15), 3.0(.99)$, respectively; $F(2,145)=1.15, p=0.32$ ) by risk/treatment group for adolescents. Similarly no differences were found for psychosocial treatment willingness by risk/treatment group for parents $(M(S D)$ of 4.1 (.90), 3.9 (.64), 3.8 (1.02), respectively; $F$ $(2,158)=1.85, p=0.16)$. However, parental medication willingness varied by risk/treatment group status $(M(S D)$ of $3.5(1.21), 2.4(1.20), 3.0(1.26)$, respectively; $F(2,157)=8.04, p<$. 001 ), and pairwise comparison using the Tukey-Kramer adjustment showed that parents whose adolescents had been previously treated for ADHD expressed higher willingness than those parents whose high-risk adolescent had never been treated $(\mathrm{M}=3.44$ versus $2.41, p<$. 0004). 


\section{Qualitative methods and findings}

In this study, we applied grounded theory methods to analyze open-ended survey responses to the question "what other undesirable effects are you concerned about?" Since its original formulation, grounded theory has been applied to diverse disciplines and forms of data. Even though grounded theory methods are more typically used for data which yield more extensive responses, like semi-structured interviews, our written dataset provided numerous elaborated written responses that supported constant comparison, productive detailed analysis, and theory development. Glaser (1978) defined grounded theory as "a detailed grounding by systematically analyzing data sentence by sentence by constant comparison as it is coded until a theory results" (p. 16) (Glaser, 1978). Furthermore, in grounded theory analysis various coding levels, constant comparison, and memoing are used to ensure that the resulting theory and the conclusions drawn from the data are grounded in the data (Charmaz, 2006; Glaser \& Strauss, 1967; Strauss, 1998). More specifically, grounded theory analysis proceeds from the specific to the general by beginning with particular pieces of data (codes) and continuing toward the development of a meaningful whole (theory).

In our data participants' response lengths varied from one word to lengthy paragraphs. Of the 1,356 written responses most addressed the use of short acting (352 responses) and long acting medications (277 responses). Participants produced the fewest concerns related to psychoeducation (151 responses). Parents, teachers, and health professionals had similar written response rates, whereas adolescents provided fewer written responses. Our multidisciplinary coding team, consisting of a qualitative research methodologist (MK), a child and adolescent psychiatrist ADHD expert and services researcher (RB), a graduate student in social work (DM), and a senior undergraduate psychology student (GM). All team members were trained by the qualitative methodologist to open code, compare data and emerging codes. The research team coded survey responses based on a shared codebook and ongoing team discussions about the data content. Codebook and the emerging list of open codes were continuously discussed and revised together. Examples of open codes included codes such as loss of appetite, heart problems, isolation, unwillingness to use, future drug addiction. We followed Strauss and Corbin's (1998) subsequential steps for coding (axial coding and selective coding). During axial coding we sought to identify explicit connections between categories and sub-categories. Examples of axial codes included ineffective treatment, cost, and treatment dependence. Later we developed selective codes through constant comparison with the intention of reducing and selecting codes to develop theory (Bryant \& Charmaz, 2007; Holton, 2007). Theoretical codes were used to achieve an integrated theoretical model describing participants' general negative perceptions of ADHD treatments (negative attitude/disliking; anticipated ineffectiveness; burden) and expectations of specific undesirable effects (physiological and psychological side effects, stigma and future dependence). In our model shown in Figure 1 thick lines indicate larger coding categories ( 20 or more responses) and thus also stronger connections between theoretical codes and study interventions. Axial codes of theoretical codes are indicated through numerical signs. For example, "Burden" included considerations of cost (axial code 3 ), disruption (axial code 4), up to required consistency (axial code 9). We also investigated how codes were distributed across respondent types. We did not find any large representation clusters, with two minor exceptions. Both teachers and school professionals were concerned about short term medication disrupting academics and learning. Additionally, both school and health professionals linked short term medication to emotional side-effects such as sadness and irritability. Generally though the identified thematic clusters and categories were mentioned by all respondents and our model applies to all participants.

Short and long acting medications-Short and long acting medications were associated with diverse general negative perceptions, but similar expectations of specific 
untoward effects. Short acting medication (but not long acting) was associated with negative attitudes and dislike. All respondent groups except health care professionals made statements that short acting medication should be combined with other interventions such as counseling, "behavior modification programs", classroom accommodations, or education that would teach students coping skills. Both short and long acting medications were described as burdensome; however, short acting medications were viewed as particularly disruptive of students' academics, learning, and school schedules. Additionally, short acting medication use was associated with stigma and feelings of embarrassment; adults voiced that teens would be "stigmatized by peers for taking meds at school".

Nevertheless, both types of medication were perceived as having identical physiological side effects, most commonly loss of appetite, weight loss, lethargy or lack of energy, and sleeping problems (especially insomnia). Similarly, both medication types were thought to cause similar psychological side effects, resulting in children appearing sad, depressed, and irritated. Unlike long-acting medication, short acting medication was seen as creating stigma that lowered children's self-esteem. Finally, the use of both types of ADHD medication generated concerns of future dependency, especially future addiction to medications and treatment dependency.

Education about $A D H D$ was associated with the fewest general negative perceptions and no specific untoward effects. In general, participants described education about ADHD as important and as a "first step" in treatment, but they also argued "education without an action plan is not very effective by itself". Participants also illustrated their willingness to engage in educational interventions but they found education not helpful in itself. Rather, they suggested that "education about ADHD is imperative, but without complementary strategies, it will not be very helpful".

Behavior therapy and counseling-Behavior therapy and counseling elicited similar negative attitudes and dislike. For example, one teenager viewed ADHD as "a medical problem they can't help so using rewards is kind of treating them like animals?" Additionally, behavior therapy and psychotherapies were seen as ineffective in general and especially ineffective by themselves. Individual counseling was seen as especially ineffective. Responses included statements like "ADHD symptoms may not improve much" or "I don't feel classroom behavior, self-control or hyper activity will properly be addressed". Many teachers saw the importance of therapy and behavior modification but at the same time they advocated for combining therapies with medical interventions.

Even though both behavior and counseling were seen as burdensome the type of burden varied. Counseling was seen as costly and stigmatizing. Some participants were worried about the "stigmatizing effects of getting counseling" stating that "public knowledge would be devastating". The biggest implementation burden for behavior therapy was the need for consistency at all levels (within the family and across different care and educational contexts) in order for it to be effective.

The only untoward effect related to counseling was increased stigma. One parent noted that stigma becomes evident when "many application forms ask if your child has ever received counseling". In contrast, behavior therapy was not viewed to be as stigmatizing as counseling; it was seen as a form of intervention that can increase conflicts between parents and teenagers and thereby escalate teens' rebellious attitudes. Parents described how behavior therapy would be "setting up a more adversarial and controlling relationship between parents and child". Additionally, all participant groups except health care providers noted how behavior therapy might create reward dependence instead of teaching teenagers to control their behavior independently, thus moving teenagers toward extrinsic motivations. 
Adults expressed concerns that teenagers would be "only performing the desired behavior for a reward. Once the reward system is removed, bad behaviors return".

\section{Discussion}

Study findings suggest that adolescents' willingness to seek ADHD treatment diverges significantly from that of adults involved in the ADHD treatment process. Adolescents were significantly less willing than adult respondents to consider medications or psychosocial treatments for ADHD. Adolescents' low willingness to use evidence-based ADHD treatments, paired with age-specific expectations to master school work with less parental involvement (Williamson et al., 2009), can cause significant academic problems for teens with ADHD. They may experience significant ADHD symptoms with resulting problems organizing, completing and handing in their school work, yet no longer receive scaffolding academic support from their parents or formal ADHD treatment. ADHD has been linked to impaired academic outcomes in various studies (Bussing et al., 2010; Frazier et al., 2007), however, it remains unclear to what degree successful adolescent ADHD treatment engagement could improve these outcomes.

Eliciting adolescents' views on ADHD treatment seems important, yet there is limited guidance for clinicians on how to implement such assessments, and what the roles of youth, parents and clinicians should be in the process. ADHD treatment guidelines for children and adolescents emphasize information exchange among adults involved in the ADHD treatment process (American Academy of Pediatrics, 2001; Pliszka, 2007). Clinicians are directed to conduct parent interviews and obtain standardized symptom ratings from parents and teachers, whereas youth are considered unreliable informants of their ADHD symptoms. Recent studies have begun to examine participatory or shared decision making approaches to ADHD in primary care settings (Fiks et al., 2010; Fiks et al., 2011) and point out important differences as to what shared or participatory decision making may mean to each party. Most notably, whereas families viewed shared decision making as a partnership between equals, clinicians understood it as a means to get families to accept the clinician's preferences (Fiks et al., 2011). We interpret our study findings to suggest that clinicians should actively communicate with both adolescents and their caregivers during the ADHD treatment planning process, because adolescents' views may differ significantly from that of their parents/caregivers. Unexpressed adult-adolescent discrepancies in treatment willingness may contribute to adolescent treatment drop-out. When seeking input on ADHD care, clinicians should consider that adolescents prefer self-reliance over formal treatment according to recent reviews of barriers and facilitators to mental health help-seeking (Gulliver et al., 2010), thus there may be no consensus on mutually agreeable interventions during a hurried office visit. The question whose values and treatment preferences should ultimately prevail in adolescent ADHD treatment planning raises complex consideration that defies simple answers (Parens \& Johnston, 2009).

Against our initial hypothesis we did not find any differences in willingness to use medications by race/ethnic background. Previous prescription database studies have documented lower rates of use of psychotropic medications, including ADHD medications, among African American populations (Zito et al., 1998). Our findings may indicate that in the context of clearly spelled out ADHD symptoms and related impairments, African American respondents apply similar considerations towards ADHD medications as Caucasian respondents, and that the differences in medication utilization noted in Medicaid studies may reflect differences in the ADHD symptom recognition process (Bussing et al., 2003) rather than differences in treatment willingness. Further studies are indicated to better understand how racial disparities in ADHD treatment utilization arise and get perpetuated. 
As hypothesized, treatment willingness was increased by certain perceptions, including feeling knowledgeable about a treatment and expectations of effectiveness, and lowered by anticipation of side-effects. For purposes of designing interventions that increase treatment engagement and lower drop-out risk, these variables may be mutable through educational interventions (Levesque et al., 2007). For example, emphasizing specific effects of ADHD treatments on attention span, completion of classroom assignments, or reduction of aggressive behavior may increase treatment willingness. Against our expectation, perceptions of embarrassment associated with a treatment did not lower treatment willingness in our survey study, even though the qualitative analysis identified stigma as a relevant side-effect concern. Our qualitative results are supported by recent reviews of barriers to adolescents' mental health help-seeking (Gulliver et al., 2010), which identified stigma/embarrassment as a main barrier. One possible explanation for our discrepant quantitative analysis results may be that our question about "embarrassment" was not a fitting proxy for the stigmatizing experiences that have been shown to be associated with ADHD and its treatment (Martin et al., 2007). The concept of stigma has multiple facets and more sophisticated measurement approaches to stigma associated with ADHD have been developed (Kellison et al., 2010; Pescosolido, 2007); however, due to concerns about respondent burden we had selected a simple stigma indicator that may not have been sufficiently sensitive.

As hypothesized, we found that past experience with ADHD treatment increased parental medication willingness, consistent with previous studies (Stroh et al., 2008). However, against our expectation, adolescents' previous ADHD treatment experience was not associated with notable differences in treatment willingness for any treatment modality. We offer several possible explanations for the absent relationship between adolescents' past ADHD treatment experience and treatment willingness. For one, the adolescent may have been uninvolved or unaware of what ADHD treatment consisted of when they were younger because the participation of children tends to be limited (Honeycutt et al., 2005); thus children may not form a cohesive conceptual memory of the treatment experience. Secondly, adolescence is a time of consolidating self-identity during which perspectives on past experiences may shift back and forth between illness and wellness, rather than following a progression of adaptation consistent with a chronic illness model (Williamson et al., 2009). Future studies should address how childhood treatment experiences influence adolescents' treatment willingness for chronic disorders like ADHD in order to inform educational interventions for children with ADHD and guide participatory decision-making efforts.

Our qualitative analysis contributed significant insights to the perceptions of side-effects related to ADHD treatments. For one, the selective codes confirmed the importance of constructs selected for the quantitative data, namely, acceptance/liking/disliking and effectiveness/ineffectiveness of a treatment modality, and emphasized the importance of a treatment burden construct, which we had not elicited through quantitative survey questions. While significant inquiry has already focused on the burden of illness (Minkoff, 2009), burden associated with treatment appears to be an important area of further study. Participants attributed significant risks not only to medications, but also to psychological interventions, and voiced concerns about immediate as well as potential future side-effects. Due to the well-publicized side effects of ADHD medications, such as growth suppression and hallucinations, health professionals may focus on what they consider most obvious, not expecting that psychosocial treatments can provoke significant side effect worries, and may therefore fail to identify relevant treatment barriers.

Our study findings need to be interpreted in the context of several important limitations, most notably sampling, geographic, and participation rates. Our sample is representative of a 
school district in a Southeastern US state, and due to school district demographic characteristics, includes only Caucasian and African American adolescents; this precludes conclusions about other racial/ethnic groups. Findings are further limited by participation rates, even though they exceed those of many other available surveys of parents, adolescents, health professionals and teachers. Because our survey method did not allow further probing, the qualitative data analysis was limited by occasional brevity of responses and by our inability to clarify or elaborate on responses with participants. Even though most of the open-ended survey responses were well crafted, and many participants wrote sentences or whole paragraphs, the interpretation of some responses could have benefitted from better contextualization and additional background information.

Despite limitations, our study contributes important new findings to the emerging literature on treatment perceptions surrounding commonly used ADHD treatments. Our multiperspective design shows significant discrepancies between adolescents' and adults' willingness to use common ADHD interventions, with low adolescent willingness for psychopharmacological and for psychotherapeutic interventions. Findings highlight the need to develop effective treatment engagement practices for adolescents with ADHD.

\section{Acknowledgments}

The authors thank the parents, adolescents, teachers and health professionals whose participation made the study possible and Dolores Albarracin, Ph.D., for consultation on the survey development. This study was supported by grant RO1MH57399 from the National Institute of Mental Health.

\section{References}

American Academy of Child and Adolescent Psychiatry. Practice Parameters for the Use of Stimulant Medications in the Treatment of Children, Adolescents, and Adults. Journal of the American Academy of Child and Adolescent Psychiatry. 2002; 41:26S-49S. [PubMed: 11833633]

American Academy of Pediatrics. Clinical Practice Guideline: Treatment of the School- Aged Child with Attention-Deficit/Hyperactivity Disorder. Pediatrics. 2001; 108:1033-1044. [PubMed: 11581465]

American Psychiatric Association. Diagnostic and Statistical Manual of Mental Disorders. 4. Washington, D.C: American Psychiatric Association; 2000. Text Revision (DSM-IV-TR)

Ascher BH, Farmer EMZ, Burns BJ, Angold A. The Child and Adolescent Services Assessment (CASA): Description and psychometrics. Journal of Emotional \& Behavioral Disorders. 1996; 4:12-20.

Bryant, A.; Charmaz, K. The SAGE handbook of grounded theory. London: SAGE; 2007.

Burns, BJ.; Angold, A.; Magruder-Habib, K.; Costello, EJ.; Patrick, MKS. The Child and Adolescent Services Assessment (CASA). Durham, N.C: Duke Medical Center; 1992.

Bussing R, Mason D, Bell L, Porter P, Garvan C. Adolescent Outcomes of Childhood AttentionDeficit/Hyperactivity Disorder in a Diverse Community Sample. Journal of the American Academy of Child and Adolescent Psychiatry. 2010; 49:595-605. [PubMed: 20494269]

Bussing R, Mason DM, Leon CE, Sinha K. Agreement between CASA parent reports and provider records of children's ADHD services. Journal of Behavioral Health Services Research. 2003; 30:462-469. [PubMed: 14593669]

Bussing R, Zima BT, Gary FA, Garvan CW. Barriers to detection, help-seeking, and service use for children with ADHD symptoms. Journal of Behavioral Health Services Research. 2003; 30:176189. [PubMed: 12710371]

Charach A, Charach A, Skyba A, Cook L, Antle BJ. Using Stimulant Medication for Children with ADHD: What Do Parents Say? A Brief Report. Journal of the Canadian Academy of Child and Adolescent Psychiatry/Journal de l'Academie canadienne de psychiatrie de l'enfant et de l'adolescent. 2006; 15:75-83. 
Charmaz, K. Constructing grounded theory: A practical guide through qualitative analysis. London: Sage; 2006.

Curtis DF, Pisecco S, Hamilton RJ, Moore DW. Teacher Perceptions of Classroom Interventions for Children with ADHD: A Cross-Cultural Comparison of Teachers in the United States and New Zealand. School Psychology Quarterly. 2006; 21:171-196.

dosReis S, Zito JM, Safer DJ, Soeken KL, Mitchell JW Jr, Ellwood LC. Parental Perceptions and Satisfaction with Stimulant Medication for Attention-Deficit Hyperactivity Disorder. Developmental and Behavioral Pediatrics. 2003; 24:155-162.

Fiks AG, Gafen A, Hughes CC, Hunter KF, Barg FK. Using freelisting to understand shared decision making in ADHD: Parents' and pediatricians' perspectives. Patient Education and Counseling. 2010

Fiks AG, Hughes CC, Gafen A, Guevara JP, Barg FK. Contrasting parents' and pediatricians' perspectives on shared decision-making in ADHD. Pediatrics. 2011; 127:e188-196. [PubMed: 21172996]

Frazier TW, Youngstrom EA, Glutting JJ, Watkins MW. ADHD and Achievement: Meta-Analysis of the Child, Adolescent, and Adult Literatures and a Concomitant Study With College Students. Journal of Learning Disabilities. 2007; 40:49-65. [PubMed: 17274547]

Frisch L, Moser SE, Hawley F, Johnston J, Romereim M. Kansas School Nurses' knowledge and opinions about ADHD stimulant medication therapy. (Health Service Applications).(AttentionDeficit/Hyperactivity Disorder). Journal of School Health. 2003; 73(127):122.

Gage JD, Wilson LJ. Acceptability of attention-deficit/hyperactivity disorder interventions: A comparison of parents. Journal of Attention Disorders. 2000; 4:174-182.

Glaser, B. Theoretical sensitivity. Mill Valley, CA: The Sociology Press; 1978.

Glaser, B.; Strauss, A. The discovery of grounded theory. Chicago: Aldine Publishing Company; 1967.

Gulliver A, Griffiths KM, Christensen H. Perceived barriers and facilitators to mental health helpseeking in young people: a systematic review. BMC Psychiatry. 2010; 10:113. [PubMed: 21192795]

Hansen DL, Hansen EH. Caught in a Balancing Act: Parents' Dilemmas Regarding Their ADHD Child's Treatment with Stimulant Medication. Qualitative Health Research. 2006; 16:1267-1285. [PubMed: 17038757]

Hoagwood K, Kelleher KJ, Feil M, Comer DM. Treatment services for children with ADHD: a national perspective. Journal of the American Academy of Child and Adolescent Psychiatry. 2000; 39:198-206. [PubMed: 10673831]

Hollingshead, AB. Four Factor Index of social class. Yale University, Department of Sociology; 1975.

Holton, J. The coding process and its challenges. In: Bryant, A.; Charmaz, K., editors. The SAGE handbook of grounded theory. London: SAGE; 2007. p. 265-290.

Honeycutt C, Sleath B, Bush PJ, Campbell W, Tudor G. Physician use of a participatory decisionmaking style with children with ADHD and their parents. Patient Education and Counseling. 2005; 57:327-332. [PubMed: 15893216]

Johnston C, Hommersen P, Seipp C. Acceptability of Behavioral and Pharmacological Treatments for Attention-Deficit/Hyperactivity Disorder: Relations to Child and Parent Characteristics. Behavior Therapy. 2008; 39:22-32. [PubMed: 18328867]

Kellison I, Bussing R, Bell L, Garvan C. Assessment of stigma associated with attention-deficit hyperactivity disorder: psychometric evaluation of the ADHD stigma questionnaire. Psychiatry Research. 2010; 178:363-369. [PubMed: 20580842]

Krain AL, Kendall PC, Power TJ. The role of treatment acceptability in the initiation of treatment for ADHD. J Atten Disord. 2005; 9:425-434. [PubMed: 16371665]

Levesque P, Davidson S, Kidder K. Knowledge exchange for Attention Deficit Hyperactivity Disorder Research: an integrated evidence and knowledge exchange framework leading to more effective research dissemination practices. Journal of the Canadian Academy of Child and Adolescent Psychiatry. 2007; 16:51-56. [PubMed: 18392152]

Martin JK, Pescosolido BA, Olafsdottir S, McLeod JD. The construction of fear: Americans' preferences for social distance from children and adolescents with mental health problems. Journal of Health and Social Behavior. 2007; 48:50-67. [PubMed: 17476923] 
McNeal RE, Roberts MC, Barone VJ. Mothers' and Children's Perceptions of Medication for Children with Attention-Deficit Hyperactivity Disorder. Child Psychiatry and Human Development. 2000; 30

Minkoff NB. ADHD in managed care: an assessment of the burden of illness and proposed initiatives to improve outcomes. American Journal of Managed Care. 2009; 15:S151-159. [PubMed: 19601690]

Moline S, Frankenberger W. Use of Stimulant Medication for Treatment of Attention-Deficit Hyperactivity Disorder: A Survey of Middle and High School Students' Attitudes. Psychology in the Schools. 2001; 38:569-584.

Parens E, Johnston J. Facts, values, and attention-deficit hyperactivity disorder (ADHD): an update on the controversies. Child and Adolescent Psychiatry and Mental Health. 2009; 3:1. [PubMed: 19152690]

Pelham WE Jr, Fabiano GA. Evidence-based psychosocial treatments for attention-deficit/ hyperactivity disorder. Journal of Clinical Child Adolescent Psychology. 2008; 37:184-214.

Pescosolido BA. Culture, children, and mental health treatment: special section on the national stigma study-children. Psychiatric Services. 2007; 58:611-612. [PubMed: 17463339]

Pliszka S. Practice parameter for the assessment and treatment of children and adolescents with attention-deficit/hyperactivity disorder. Journal of the American Academy of Child and Adolescent Psychiatry. 2007; 46:894-921. [PubMed: 17581453]

Polanczyk G, de Lima MS, Horta BL, Biederman J, Rohde LA. The Worldwide Prevalence of ADHD: A Systematic Review and Metaregression Analysis. American Journal of Psychiatry. 2007; 164:942-948. [PubMed: 17541055]

Power TJ, Hess LE, Bennett DS. The acceptability of interventions for attention-deficit hyperactivity disorder among elementary and middle school teachers. Journal of Developmental and Behavioral Pediatrics. 1995; 16:238-243. [PubMed: 7593658]

Rey JM, Sawyer MG. Are psychostimulant drugs being used appropriately to treat child and adolescent disorders? The British Journal of Psychiatry. 2003; 182:284-286. [PubMed: 12668399]

Stockl KM, Hughes TE, Jarrar MA, Secnik K, Perwien AR. Physician perceptions of the use of medications for attention deficit hyperactivity disorder. Journal of Managed Care Pharmacy. 2003; 9:416-423. [PubMed: 14613439]

Strauss, A.; Corbin, J. Basics of qualitative research: techniques and procedures for developing grounded theory. Thousand Oaks: Sage; 1998.

Stroh J, Frankenberger W, Cornell-Swanson LV, Wood C, Pahl S. The use of stimulant medication and behavioral interventions for the treatment of attention deficit hyperactivity disorder: A survey of parents' knowledge, attitudes, and experiences. Journal of Child and Family Studies. 2008; 17:385-401.

Vereb RL, DiPerna JC. Teachers' Knowledge of ADHD, Treatments for ADHD, and Treatment Acceptability: An Initial Investigation. School Psychology Review. 2004; 33:421-428.

Williamson P, Koro-Ljungberg ME, Bussing R. Analysis of critical incidents and shifting perspectives: transitions in illness careers among adolescents with ADHD. Qualitative Health Research. 2009; 19:352-365. [PubMed: 19224878]

Zito JM, Safer DJ, dosReis S, Riddle MA. Racial disparity in psychotropic medications prescribed for youths with Medicaid insurance in Maryland. Journal of the American Academy of Child and Adolescent Psychiatry. 1998; 37:179-184. [PubMed: 9473914] 


\section{Research highlights}

- There are significant discrepancies among US teens', parents', teachers' and health professionals' willingness to use ADHD interventions.

- Teens express low willingness for psychopharmacological and psychotherapeutic ADHD treatments.

- Expectations of Treatment burden and fear of fostering reward dependence or drug addiction lower ADHD intervention willingness. 


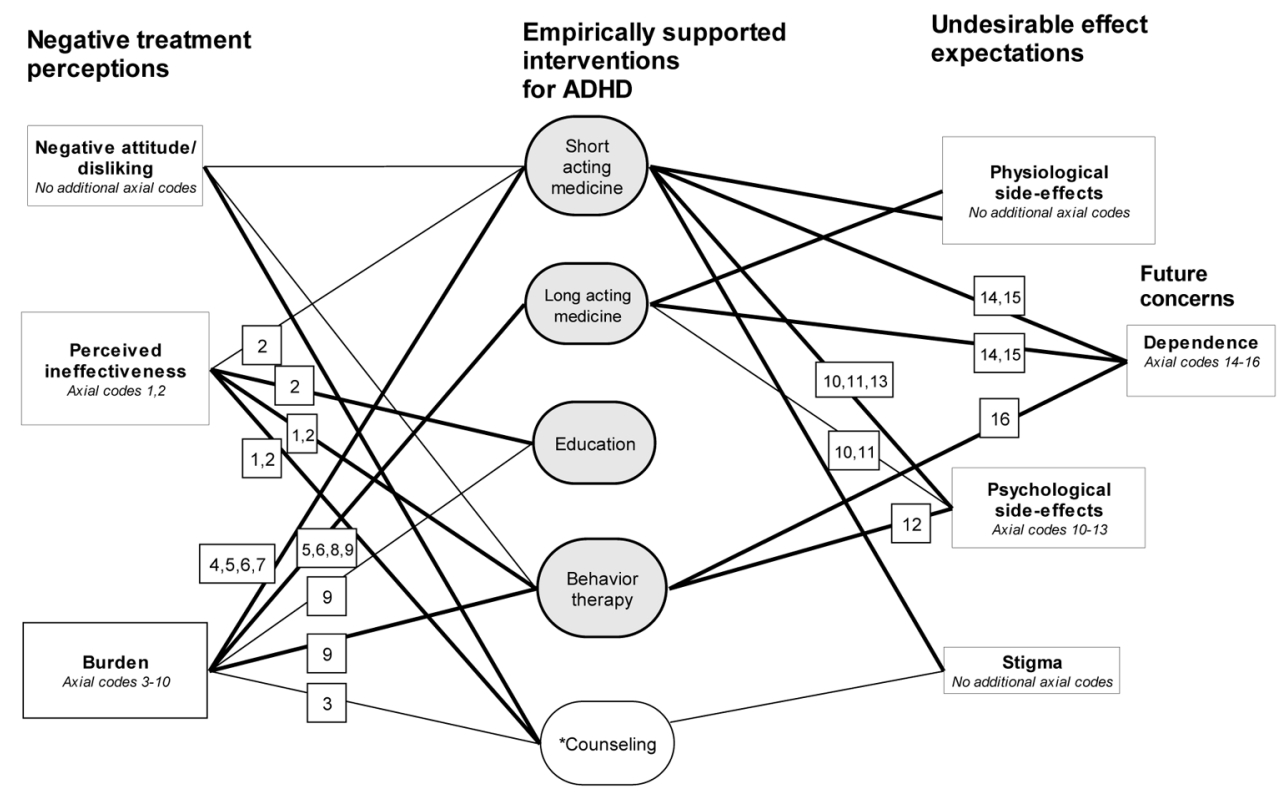

Figure 1.

Undesirable effects of empirically supported ADHD interventions and of counseling Legend of axial codes as described by adolescents, parent, teachers, and health providers: Selective code of perceived ineffectiveness of treatment- Axial codes: 1= Ineffective treatment, Axial code $2=$ Ineffective treatment until combined with other treatments Selective code of burden of treatment- Axial codes: $3=$ Cost, $4=$ Disruption of daily activities, $5=$ Forgetting to take medicine, $6=$ Dosing concerns, $7=$ Medication wears off prematurely, $8=$ Supervision needed to follow the treatment regimen, $9=$ Treatment requires consistency

Selective code of psychological side-effects- Axial codes: 10= Sadness, depression, $11=$ Irritability, 12= Increased conflict, 13= Low self-esteem

Selective code of dependence-Axial codes: 14= Medicine addiction, $15=$ Treatment dependence, 16= Reward dependence

*Counseling is not categorized as an empirically supported ADHD treatment (Pelham \& Fabiano, 2008) Note: A thick line indicates more than 20 participant responses 


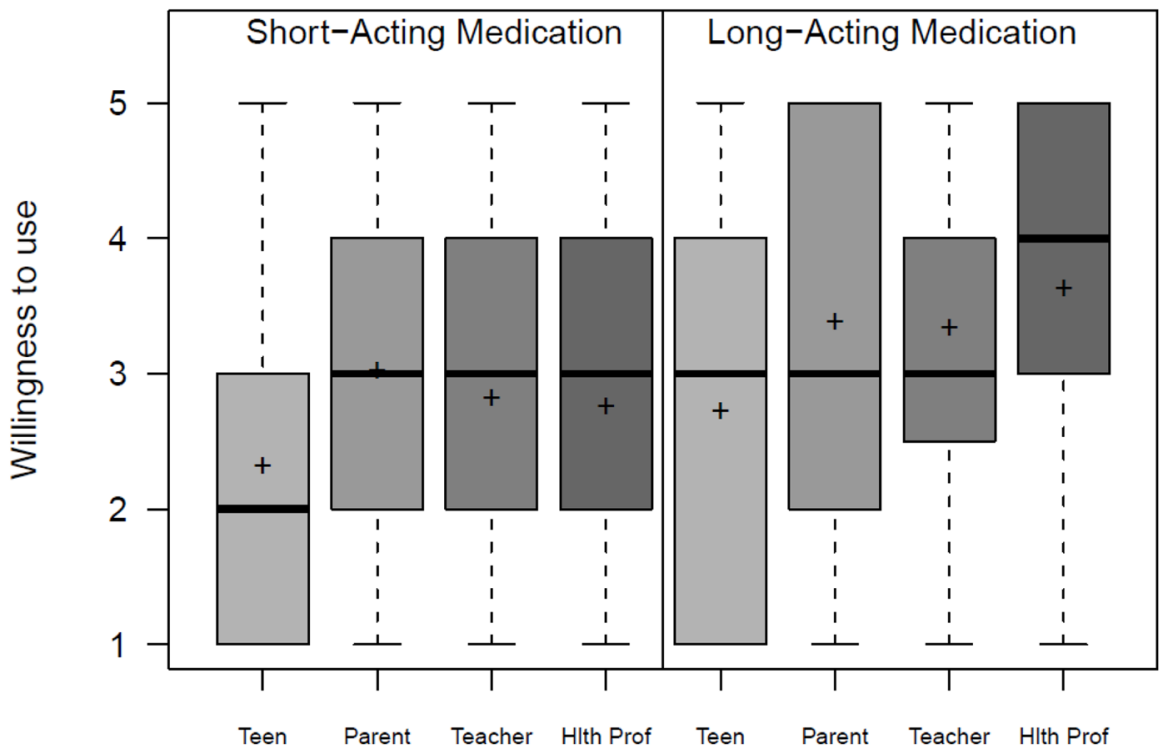

Graph 1.

Willingness to use medications 


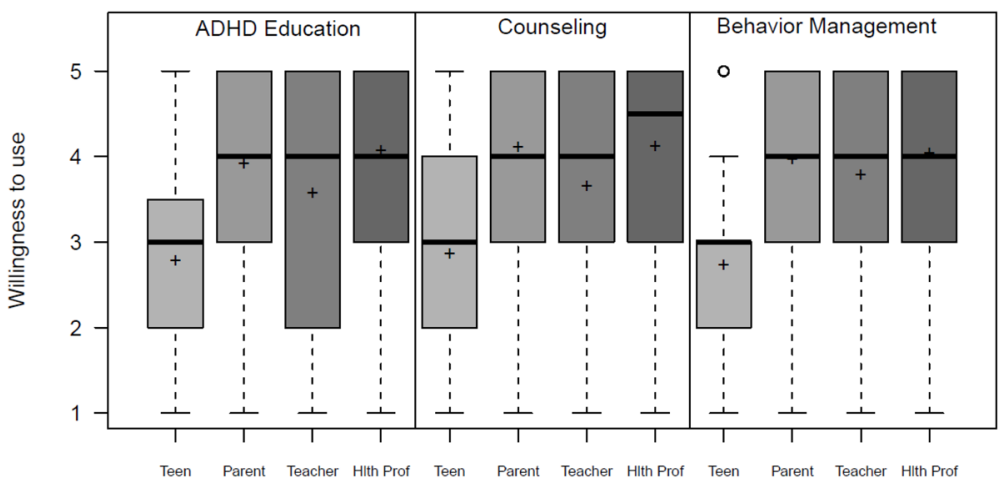

Graph 2.

Willingness to use psychosocial treatments 


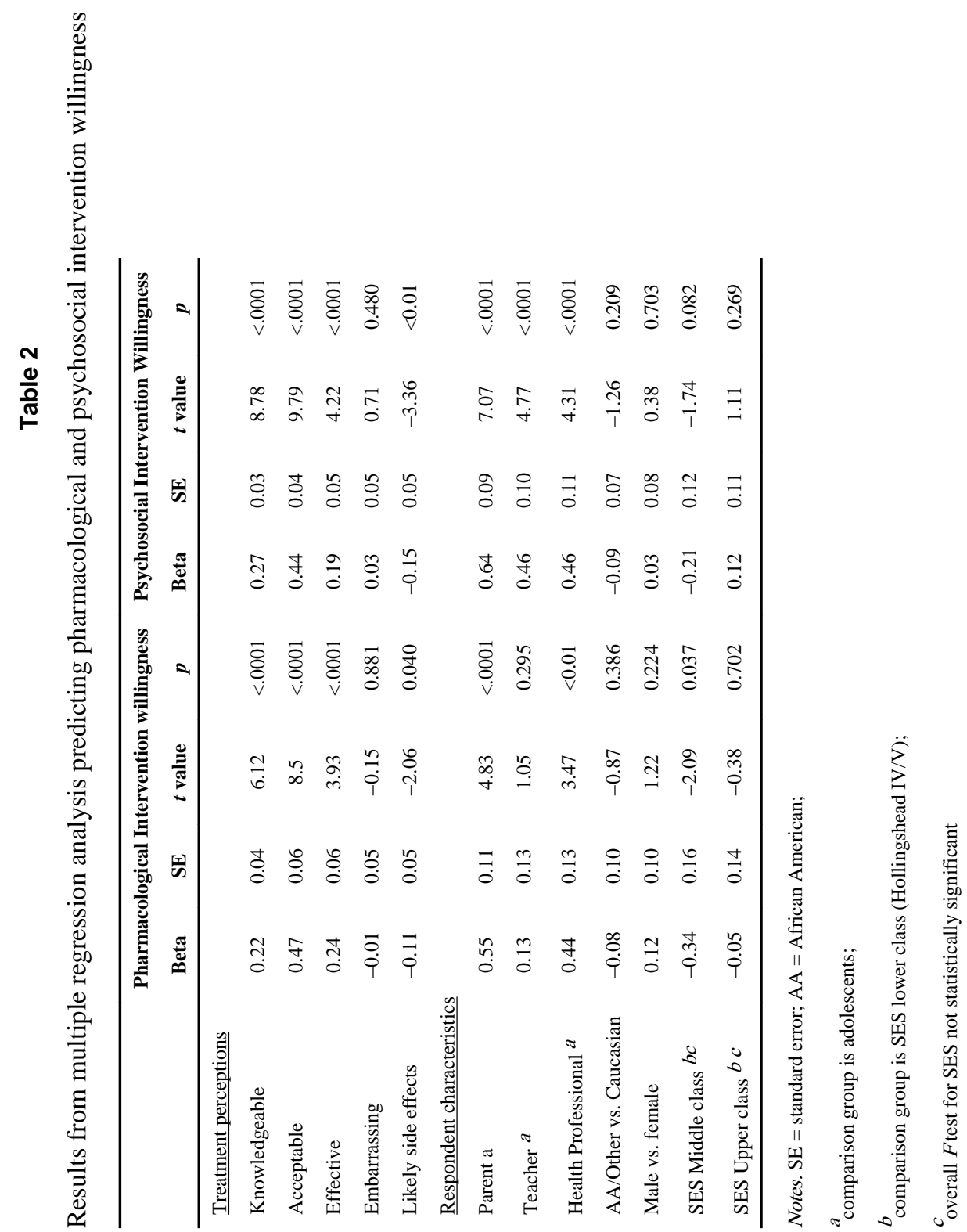

